Cite this: Phys. Chem. Chem. Phys., 2014, 16, 7368

Received 26th November 2013, Accepted 21st February 2014

DOI: $10.1039 / \mathrm{c} 3 \mathrm{cp} 54986 \mathrm{k}$

www.rsc.org/pccp

\title{
$A b$ initio quantum mechanical simulations confirm the formation of all postulated species in ionic dissociation
}

\author{
Martin J. Wiedemair, ${ }^{a}$ Alexander K. H. Weiss ${ }^{b}$ and Bernd M. Rode*a
}

\begin{abstract}
A single sodium chloride molecule in aqueous solution was simulated by the ab initio quantum mechanical charge field-molecular dynamics (QMCF-MD) approach. During a series of simulations the solvated molecule (CIP), dissociated solvated ions and - most noticeably - a solvent separated ion pair (SSIP) were observed and the structural and dynamical characteristics of these systems were investigated. In addition to a detailed structural analysis of the observed species, vibrational spectra and charge distributions were calculated to elucidate the mechanism of the $\mathrm{NaCl}$ dissociation.
\end{abstract}

\section{Introduction}

General experience tells us that many salts, such as sodium chloride, dissociate in water, but the exact mechanism of how this happens largely remains a matter of hypothesis, owing to the fact that this process occurs on the picosecond time scale and is, therefore, hardly accessible via experimental methods. By applying computational simulation methods one can get an impression of how such a mechanism might proceed, provided that the simulations include relevant contributions to appropriately describe all atomic interactions. Recent studies prove the continuous relevance of this problem, employing either experimental $^{1,2}$ or theoretical approaches. ${ }^{1-10}$

Most of the research studies conducted to date are molecular mechanics approaches utilizing different force fields. In an article published in 2006 Bouazizi et al. ${ }^{1}$ compared MD simulations of different concentrations of $\mathrm{NaCl}_{(\mathrm{aq})}$ with experimental X-ray scattering results and reported reasonable results. Smith and Dang $^{11}$ outlined the importance of using polarizable water models when dealing with such systems. They focused their work on the CIP and SSIP species as first suggested by Winstein. ${ }^{12}$ Fennell and coworkers ${ }^{9}$ expanded the field by conducting a comparative study to analyze all possible alkali-halide ion pairs employing different force fields and water models. They concluded that although the general trend was retained, the individual results were strongly dependent on the used force field. A similar conclusion can be drawn from the studies of Patra and Karttunen. ${ }^{3}$ Their systematic comparison of force fields for microscopic simulations of aqueous $\mathrm{NaCl}$ was based on a $0.87 \mathrm{M}$ solution and six commonly used

\footnotetext{
${ }^{a}$ Department of Theoretical Chemistry, University of Innsbruck, Innrain 80-82, Innsbruck, Austria. E-mail: bernd.m.rode@uibk.ac.at

${ }^{b}$ University of Munich, Munich, Germany
}

force fields (Gromacs, X-Plor/Charmm-22, Charmm-27, Amber1999, OPLS-AA and Smith-1994) combined with 4 well-established water models (SPC, SPC/E, TIP3P, TIP4P). These calculations produced widely diverging results for the solution properties, depending on the force field and the water model used. In some simulations even $\mathrm{NaCl}$ clusters were formed, leading to the conclusion that in $0.87 \mathrm{M}$ aqueous solution $\mathrm{NaCl}$ would eventually precipitate at room temperature. Clustering effects are reported, however, only for supercritical conditions. ${ }^{8}$ The results of these studies indicate that the majority of commonly used force fields are inadequate to describe the chemistry of ionic systems in solution. Recent investigations conducted by Timko and his research team were already dedicated to finding appropriate force constants for the ion-ion interaction in aqueous $\mathrm{NaCl}$ solution by applying $a b$ initio methods. Furthermore, they explicitly acknowledge the formation of a solvent separated ion pair. ${ }^{5}$ Geissler et al. provided an interesting look into the complexity of an ionic dissociation and emphasized the importance of including explicit solvent dynamics. ${ }^{4}$ One of the few quantum mechanical approaches to this topic was taken up by Ghosh et al. ${ }^{13}$ In their investigation of $\mathrm{NaCl}\left(\mathrm{H}_{2} \mathrm{O}\right)_{1-6}$ clusters and subsequent QM/EFP-MD simulation they found and described several Interionic Hydration Structures (IHS), in addition to the CIP and SSIP species.

The aim of this work was to investigate the properties of a single $\mathrm{NaCl}$ ion pair in aqueous solution, by using the recently developed Quantum Mechanical Charge Field Molecular Dynamics approach (QMCFMD). ${ }^{14,15}$ The $\mathrm{NaCl}_{\mathrm{aq}}$ system can be considered as the first step in dissociation and, since the quantum chemical treatment does not employ any predetermined parameters to describe the interaction, properties such as the effective bond strength of the $\mathrm{NaCl}$ pair and the mechanism of dissociation of the molecule in water can be obtained very accurately, and without any bias. The respective results will contribute significantly to 
the understanding of the structural and dynamical processes accompanying dissociation at a molecular level and will thus aid researchers in the construction of more accurate force field parametrizations for ions in solution.

\section{Methods}

\subsection{Simulation method}

As mentioned before, the Quantum Mechanical Charge Field Molecular Dynamics (QMCFMD) method ${ }^{14-16}$ was used for this simulation. In conventional quantum mechanics/molecular mechanics (QM/MM) methods, ${ }^{17-19}$ the simulation box is divided into two separate parts. The QMCF-MD method enhances this conventional methods by adding a QM solvation layer, thereby increasing the volume described by the QM calculation significantly. Furthermore, it is based on the assumption that the distance between particles in the central region of the QM-zone, termed the core region, and the MM zone is beyond the nonCoulombic cutoff distance of typical potentials. Therefore, only Coulombic interactions - plus a correction term to account for the use of a Coulombic cutoff - have to be considered to describe the interactions of particles in the core-zone with the atoms located in the MM region. Further details on the QMCF approach are given elsewhere. ${ }^{14-16,20}$ Since the aim of this study was to specifically analyze the properties and the dissociation behavior of $\mathrm{NaCl}$ in water, different simulations were carried out, centering the QM region on one of the two ions $\left(\mathrm{Na}^{+}\right.$or $\left.\mathrm{Cl}^{-}\right)$. Initially two simulations were set up: one centered at $\mathrm{Na}^{+}$and the other centered at $\mathrm{Cl}^{-}$. Since the $\mathrm{Na}^{+}$-centered simulation resulted in a rapid dissociation of the $\mathrm{NaCl}$ pair, a second one was carried out, employing a different starting structure. The splitting of the simulation work into two different runs was a necessary consequence of the extremely large computational effort for a single run describing all possible equilibration mechanisms. Different starting conditions enable an easier access to the various possible reactions and species encountered in this context.

\subsection{Simulation protocol}

At first an ideal core-zone starting structure, describing the $\mathrm{NaCl}$ molecule and its first hydration shell, was obtained by performing energy minimization of $\mathrm{NaCl}\left(\mathrm{H}_{2} \mathrm{O}\right)_{n}$-clusters, with $n \leq 7$, using Gaussian09. ${ }^{21}$ Surrounding this initial structure a periodic cubic simulation box with an edge length of $31.11 \AA$ was constructed, containing a total of 1000 water molecules and the $\mathrm{NaCl}$ molecule. In all simulations the flexible, anharmonic SPC-mTR water model ${ }^{22,23}$ was used for the MM region, enabling explicit hydrogen movements required for a smooth transition of molecules between the QM and the MM zones. The density of the system was fixed at $0.997 \mathrm{~g} \mathrm{~cm}^{-3}$, i.e. the density of pure water at $298.15 \mathrm{~K}$. All simulations conducted during this study used the Berendsen thermostat ${ }^{24}$ with a relaxation time of 0.25 ps to maintain this temperature in the simulations.

The box was equilibrated for a total of 100 ps by means of conventional MM/MD simulations. The non-Coulombic interactions were calculated by potential functions of the Lennard-Jones (6-12) type ${ }^{25}$ the parameters reported by Jorgensen and Tirado-Rives in their OPLS-force field ${ }^{26}$ were employed. After the system had been properly equilibrated, the actual QMCF computation was set up. As for all QM calculations, the choice of an appropriate basis set is a most crucial task. On the one hand, one strives to achieve a high level of accuracy, on the other hand, every gain in accuracy increases the computational effort dramatically and a suitable compromise has to be sought. For this work the Hartree-Fock level and the 6-31G (d,p) basis set ${ }^{27,28}$ were chosen, as correlation effects can be considered minor in the system investigated. ${ }^{14,29}$ The core-zone radius was set to $1 \AA$ in all simulations, thus containing only the central species. The surrounding solvation layer ranged from $1 \AA$ to $5.8 \AA$. The smoothing zone thickness was set to $0.2 \AA$ to ensure a smooth transition between the QM- and the MM-zones. Interactions within the remaining volume were accounted for via molecular mechanics. To compensate for the Coulombic cutoff the reaction field method $^{30,31}$ with a permittivity $(\varepsilon)$ of 78.0 was employed.

\subsection{Evaluation methods}

The structural properties of the system were analyzed mainly via radial distribution functions (RDF) and coordination distribution functions (CND), and the dynamics via mean residence times (MRT), $\tau$, evaluated using the direct method. ${ }^{32}$

In addition angular radial distribution functions (ARD) were computed. The vibrational power spectrum was computed via Fourier transformation of the velocity auto-correlation function (VACF) (eqn (1)).

$$
C(t)=\frac{\sum_{i}^{N_{t}} \sum_{j}^{N} \vec{v}_{j}\left(t_{i}\right) \vec{v}_{j}\left(t_{i}+t\right)}{\sum_{i}^{N_{t}} \sum_{j}^{N} \vec{v}_{j}\left(t_{i}\right) \vec{v}_{j}\left(t_{i}\right)}
$$

with $N$ and $N_{t}$ being the number of particles and time origins $t_{i}$ respectively; $v_{j}$ denotes the projected pair velocity of the system with respect to the $\mathrm{NaCl}$ distance vector. Choosing 1000 time origins, the vibrational power spectrum was obtained and the respective effective force constants $(k)$ were calculated according to eqn (2):

$$
k=(2 \pi c \bar{\lambda})^{2} \mu
$$

with $\bar{\lambda}$ being the wave number gained from the vibrational spectrum, $c$ the speed of light and $\mu$ the reduced mass of the system. In this analysis pair distributions in different core segments with respect to the $\mathrm{NaCl}$ distance vectors were computed, yielding detailed information on the solvent distribution surrounding $\mathrm{NaCl}$.

\section{Results and discussion}

\subsection{General features of the systems}

In the chloride-centered simulation the solute did not separate for more than $30 \mathrm{ps}$, while the sodium-centered system showed an entirely different behavior. The sodium-centered simulation came to an end rather quickly, since the ions dissociated after 
about $4 \mathrm{ps}$, and hence the simulation was considered complete. However, the ions did not continuously dissociate, but temporarily formed an intermediate, in which they were separated by one single water molecule binding to both ions, thus forming a solvent separated ion pair (SSIP). Due to the fast dissociation of the sodium-centered simulation a second run was started using a different starting structure, which was taken from the chloridecentered simulation after a simulation time of about 6 ps. This system gradually evolved towards a similar behavior but the solvent separated ion pair was formed for a much longer period, than in the first run. It seems to be important to mention at this point that all species postulated for electrolyte solution in the past - namely solvated molecules or contact ion pairs, solvent separated ion pairs and solvated ions ${ }^{12,33-36}$ - were identified within the picosecond scale, thus confirming the correctness of these early postulates. In the following sections details of the simulations with respect to these species and the process of dissociation will be discussed.

\subsection{Chloride-centered simulation}

Fig. 1 (top) depicts the NaCl-distance and the Mulliken partial charges $^{37,38}$ of the respective ions along the course of the simulation. Right from the beginning, it is clearly visible that such a system cannot be classified as a solvated molecule.
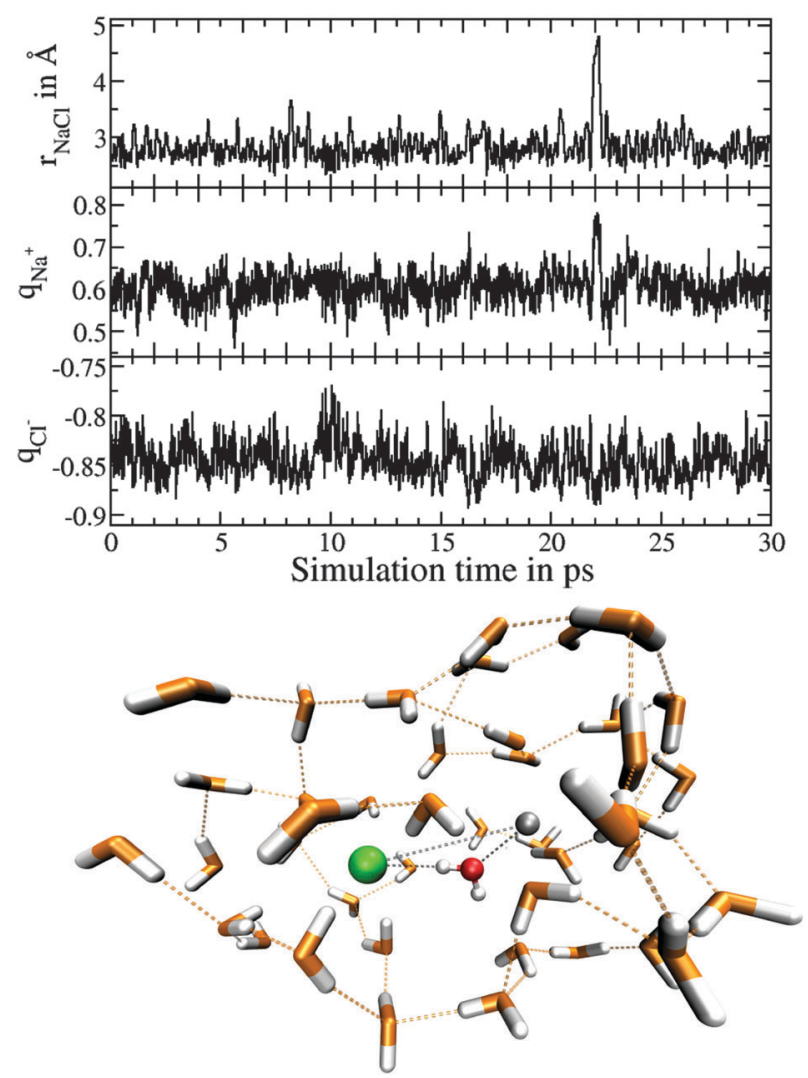

Fig. 1 Time series of the $\mathrm{NaCl}$ distance and Mulliken partial charges obtained from the chloride-centered simulation (top) and a snapshot of the simulation at 22.2 ps (bottom). The ions are separated by $4.8 \AA$, enabling a water molecule, colored in red to bind both species simultaneously.
The difference between the charges of the $\mathrm{Na}^{+}$and the $\mathrm{Cl}^{-}$ion ( +0.6 and -0.8 a.u., respectively) indicates that the term contact ion pair is a more appropriate designation. In comparison gas phase computations of a single $\mathrm{NaCl}$ pair, conducted with Gaussian $09^{21}$ at a $\mathrm{HF} / 6-31 \mathrm{G}(\mathrm{d}, \mathrm{p})$ level, yield Mulliken charges of 0.67 a.u. for sodium and chloride ions, respectively. However these results changed drastically as soon as the influence of the surrounding medium was implicitly taken into account, via a polarisable continuum model (PCM) in which case the respective charges amounted to 0.88 a.u. Neither data set matches with the situation observed in the QMCF simulation, the latter demonstrating that different charge transfer properties between the respective ions and their surrounding water molecules occur. Sodium transfers much more of its charge to the surrounding solvent molecules than chloride.

Furthermore, this plot shows that the contact ion pair reformed after a short separation of almost $5 \AA$ after just 0.4 ps (see Fig. 1, bottom; at $22.2 \mathrm{ps}$ ). As expected the charges of the $\mathrm{Na}^{+}$and $\mathrm{Cl}^{-}$ ions increase and decrease accordingly during this event, since the charge transfer between the ions is temporarily reduced. Fig. 1 depicts a snapshot of the respective configuration, revealing a water molecule binding to both ions simultaneously.

In order to further investigate the dynamics of the system, the coordination number distributions (CNDs) were calculated and the associated number of exchanges and mean residence times (MRTs) were evaluated. Table 1 summarizes these results for the first and second hydration shell of sodium and chloride, respectively. All values refer to the total simulation time of about $30 \mathrm{ps}$. $R_{\min }$ and $R_{\max }$ indicate the lower and upper shell boundary. $\mathrm{CN}_{\mathrm{av}}, N_{\mathrm{ex}}$ and $\tau_{0.5}$ denote the average coordination number, the total number of lasting exchanges $(t \geq 0.5 \mathrm{ps})$ and the mean residence time (MRT), respectively. $R_{\mathrm{ex}}$ is the reciprocal sustainability coefficient, defining how many attempts are on average required to achieve one lasting ( $t \geq 0.5 \mathrm{ps}$ ) exchange event in the given hydration sphere.

For the $\mathrm{Cl}-\mathrm{H}$ coordination number a value of 4.5 was found within the boundaries of 1.9 and $3.1 \AA$ (first shell). The coordination number distributions of $\mathrm{O}$-atoms around $\mathrm{Na}^{+}$and $\mathrm{Cl}^{-}$ions in their hydration shells are graphically presented in Fig. 2.

The obtained data (Table 1) and the two graphs (Fig. 2) demonstrate the picosecond dynamics present in this system.

Table 1 Results of the dynamical analysis of the exchange of water ligands in sodium's first and second and chloride's first hydration shell. $R_{\min }$ and $R_{\max }$ define the lower and upper shell boundary. $\mathrm{CN}_{\mathrm{av}}, N_{\text {ex }}$ and $\tau_{0.5}$ denote the average coordination number, the total number of successful exchanges and the mean residence time (MRT), respectively. $R_{\text {ex }}$ is the reciprocal sustainability coefficient, defining how many attempts are on average required to achieve one lasting ( $t \geq 0.5 \mathrm{ps}$ ) exchange event in the given hydration sphere

\begin{tabular}{llll}
\hline & $\mathrm{Na}-\mathrm{O}$ (first shell) & $\mathrm{Na}-\mathrm{O}$ (second shell) & Cl-O (first shell) \\
\hline$R_{\min } / \AA$ & 2.0 & 3.4 & 2.9 \\
$R_{\max } / \AA$ & 3.4 & 5.5 & 4.1 \\
$\mathrm{CN}_{\mathrm{av}}$ & 4.3 & 15.5 & 7.3 \\
$N_{\mathrm{ex}}$ & 40 & 327 & 142 \\
$\tau_{0.5} / \mathrm{ps}$ & 3.3 & 1.5 & 1.6 \\
$R_{\mathrm{ex}}$ & 5.2 & 5.5 & 7.1
\end{tabular}



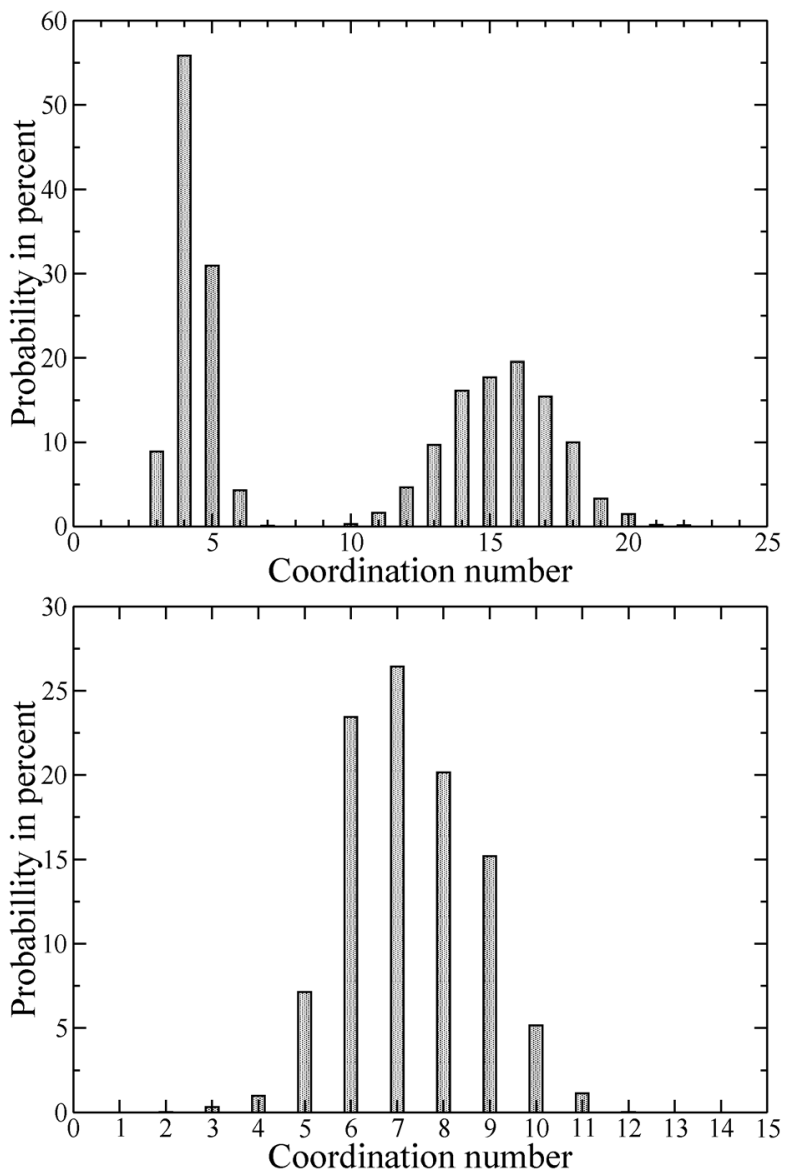

Fig. 2 Coordination Number Distributions (CNDs) of first shell oxygen around the $\mathrm{Na}^{+}$ion (top) and the $\mathrm{Cl}^{-}$ion (bottom).

The broadness of the coordination number distributions (CNDs) indicates that manifold exchange events of water molecules in the hydration layers of both ions occur. When comparing these results of the contact ion pair with simulations of the solvated, isolated ions, ${ }^{39,40}$ some noticeable differences are observed. The average first shell coordination numbers of the contact ion pair are 4.3 and 4.5 for sodium and chloride, respectively, which are lower than the values observed for the individual solvated ions amounting to 5.5 and $6{ }^{39,40}$ On the other hand, an increase in size of the first hydration spheres of both ions was observed compared to the singly solvated ions. Due to the fact that during the chloride-centered simulation the ion pair did basically not dissociate until the very end, this simulation represents one aspect of the reaction, namely the pre-dissociation phase.

The vibrational power spectrum for the $\mathrm{Na}-\mathrm{Cl}$ stretch mode obtained via velocity auto-correlation function (VCAF) and subsequent Fourier transformation is depicted in Fig. 3a (solid black curve). Close investigation of the observed peak and its shoulder leads to the conclusion that they can be decomposed by at least two Gaussian functions represented by the blue dash-dotted line and the purple, dash-double-dotted line. The dashed red line depicts the combination of the two, which coincides with the original spectrum in the region of interest.

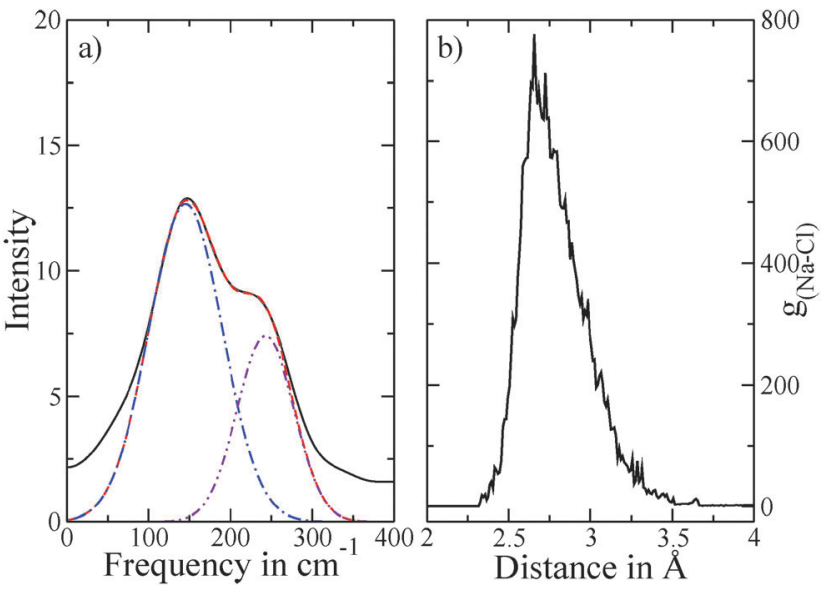

Fig. 3 (a) Vibrational power spectrum of $\mathrm{NaCl}$ with underlying Gaussian functions; (b) radial pair distribution of $\mathrm{NaCl}$.

The maxima of the two underlying Gaussians are located at 145 and $243 \mathrm{~cm}^{-1}$, respectively, the corresponding force constants are 17.3 and $48.6 \mathrm{~N} \mathrm{~m}^{-1}$. A gas phase calculation conducted with Gaussian $09^{21}$ at the HF/6-31G(d,p) level employing PCM to implicitly account for solvent effects yielded a harmonic frequency of $183 \mathrm{~cm}^{-1}$ corresponding to a force constant of $27.6 \mathrm{~N} \mathrm{~m}^{-1}$, whereas without PCM the corresponding values are $358 \mathrm{~cm}^{-1}$ and $105.6 \mathrm{~N} \mathrm{~m}^{-1}$. This finding elegantly demonstrates the strong difference between gas phase and solution and the importance of explicitly taking the surrounding solvent molecules into account.

Despite the peculiar shape of the observed vibrational power spectrum, no hint of this particular behaviour can be observed in the $\mathrm{NaCl}$ pair distribution function. However, the appearance of two peaks in the vibrational spectrum could be attributed to the coordination number of water ligands to $\mathrm{Na}^{+}$- dominantly 4 and 5 (see Fig. 2) - and a respective strengthening or weakening of the $\mathrm{NaCl}$ bond. In order to obtain structural information about the species formed, several analysis tools were used. At first the Radial Distribution Functions (RDFs) were calculated and analyzed. The data obtained by the chloride-centered simulation, with a long lifetime of the contact ion pair, provided a description of the $\mathrm{NaCl}$ bond length (Fig. 3b) with a typical NaCl-distance of $2.65 \AA$. This result is in good agreement with the results obtained from gas phase calculations mentioned in the previous section, which yielded $2.68 \AA$ for the $\mathrm{NaCl}$ distance. Spectroscopic studies of $\mathrm{NaCl}$ crystals found an average $\mathrm{NaCl}$ distance of $2.82 \AA \AA^{41,42}$ The difference in the $\mathrm{NaCl}$ distance can be explained by a weakening of the $\mathrm{NaCl}$ bond by all the other ionic interactions present in a crystal environment. The peak depicted in Fig. 3b shows distinct tailing, giving clear evidence that larger distances between the two ions are observed along the simulation. Radial distribution functions between the ions $\left(\mathrm{Na}^{+}\right.$and $\left.\mathrm{Cl}^{-}\right)$and the atoms of the water molecules $(\mathrm{H}$ and $\mathrm{O})$ were calculated employing a geometric restriction. ${ }^{20}$ A plane, perpendicular to the $\mathrm{NaCl}$ distance vector located at the respective center of mass was used to partition the system into two hemispheres (see Fig. 5). Evaluation of pair distribution in the solute exposed 

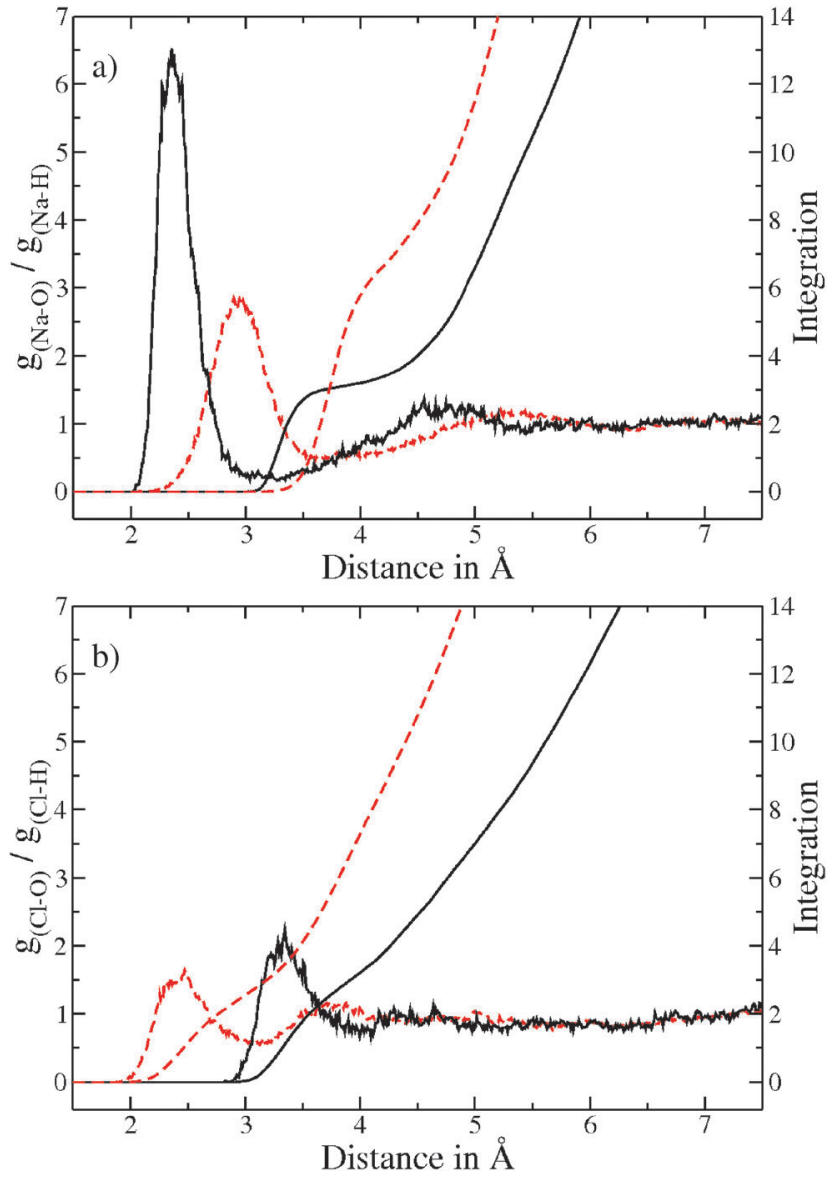

Fig. 4 (a) and (b) Radial Distribution Functions (RDFs) between $\mathrm{Na}^{+}-\mathrm{H} / \mathrm{O}$ and $\mathrm{Cl}^{-}-\mathrm{H} / \mathrm{O}$; ion-O distances are depicted as solid black, ion- $\mathrm{H}$ distances as dashed red lines.

hemisphere of the ions (the proximal hemisphere) led to the plots depicted in Fig. 4a and b.

The RDFs of $\mathrm{Na}^{+}-\mathrm{O}$ and $\mathrm{Na}^{+}-\mathrm{H}$ show that a distinct hydration structure is formed around the sodium ion. In the first layer the average $\mathrm{Na}^{+}-\mathrm{O}$ distance is about $2.3 \AA$ and the average $\mathrm{Na}^{+}-\mathrm{H}$ distance is about $3.0 \AA$. Preferential orientation of water molecules in a second sphere centered at $4.8 \AA$ is visible, but the respective peak is not very pronounced (Fig. 4a). The corresponding data of a single sodium ion in water were reported as $2.34 \AA$ A for the first and $5.0 \AA$ for the second hydration layer. ${ }^{39}$ As expected, the orientation of water molecules around the chloride ion is highly determined by hydrogen bonding, forming a rather weak hydration shell (Fig. 4b). Only one distinct hydration shell with an average distance of $3.32 \AA$ can be identified in the RDF. Existing studies denote the average $\mathrm{Cl}^{-}$-O distance as $3.15 \AA^{40}{ }^{40}$ To investigate the hydration of the $\mathrm{NaCl}$ in more detail the Angular Radial Distribution (ARD) ${ }^{43}$ was computed (Fig. 6), enabling the resolution of the structural particularities of the binding water molecules.

In Fig. 6 a distinct arrangement of water molecules around the ion pair is visible. Sodium is closely guarded by two regions in which hardly any water is exchanged, a third one can be found between them at about twice the distance. On the other hand chloride shows only one such region.

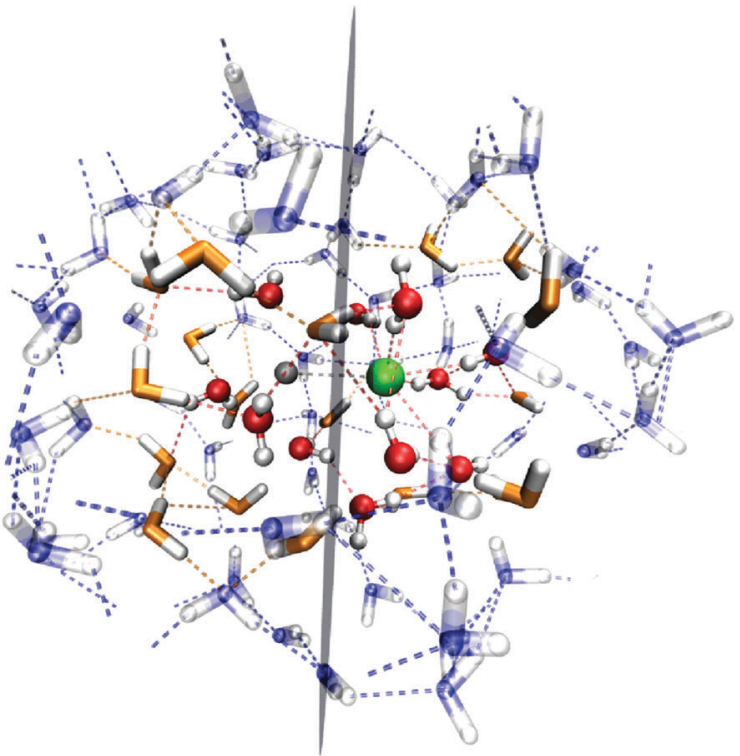

Fig. 5 Snapshot of the simulation at about 2 ps. The plane indicates the separation of the simulation box into two hemispheres, in which the RDFs were calculated respectively.

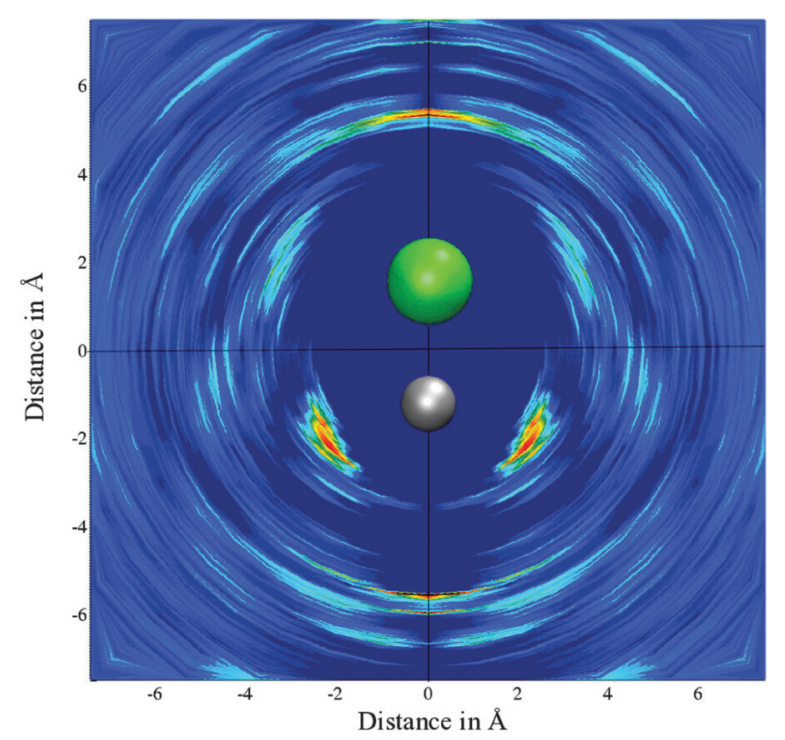

Fig. 6 Angular Radial Distribution (ARD) of water molecules around $\mathrm{Na}^{+}$ (gray sphere) and $\mathrm{Cl}^{-}$(green sphere). Red and yellow areas indicate distinct regions where water is stabilized; blue areas indicate the blur of the bulk distribution.

\subsection{Sodium-centered simulations}

3.3.1 Simulation 1. Time series of the NaCl-distance and the respective ionic partial charges observed in the first sodiumcentered simulation are depicted in Fig. 7.

In the case of the sodium partial charge plot, four distinct spikes reaching values of approximately 0.45 a.u. can be observed at 0.75 , 1.6, 2.0 and $3.5 \mathrm{ps}$. This shift of the partial charge is also observed for the chloride ion and comparison with the $\mathrm{NaCl}$ distance vector reveals that the last event occurring at 3.5 ps marks the beginning of the dissociation of the $\mathrm{NaCl}$ pair. The chloride charge plot 

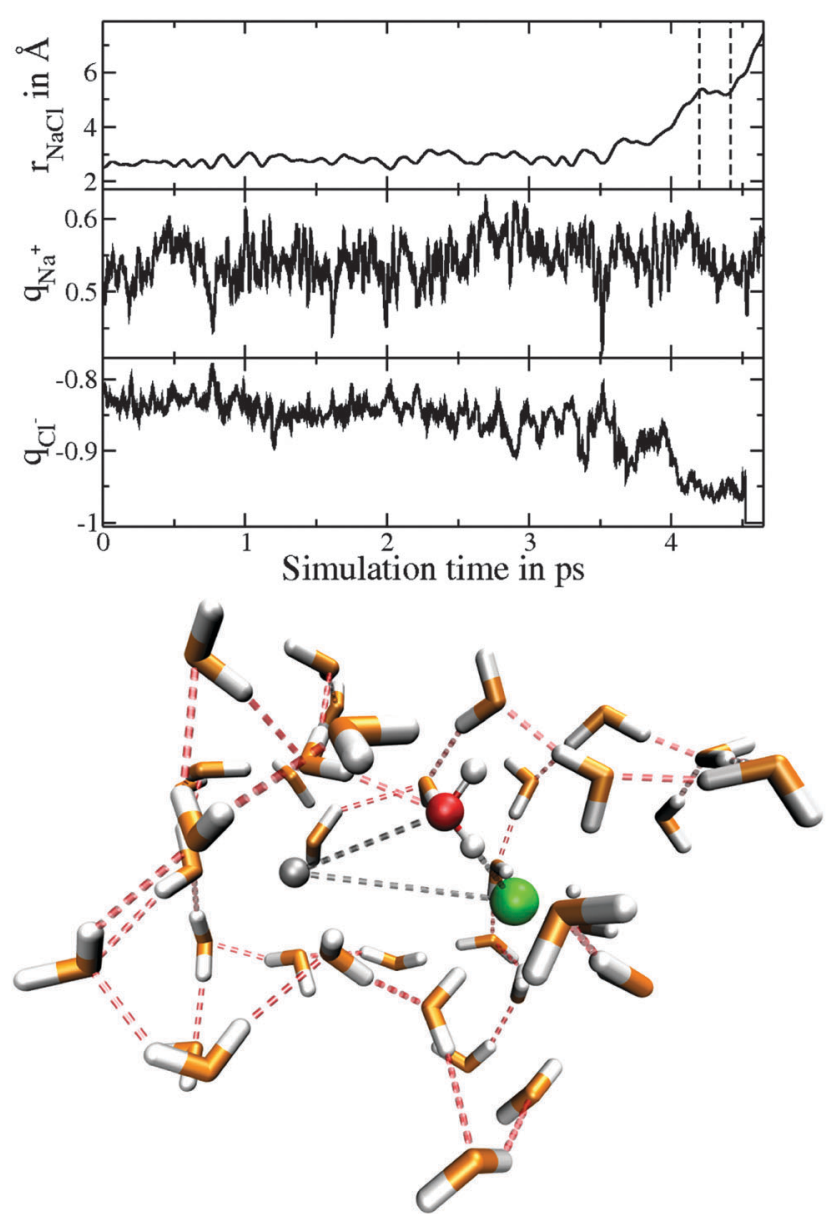

Fig. $7 \mathrm{NaCl}$ distance and respective partial charges over the course of the simulation (top) and a snapshot of the sodium-centered simulation at about 4.3 ps (bottom).

shows in several aspects a corresponding behavior. During the course of the dissociation the chloride charge decreases to -1 a.u. after about $4.5 \mathrm{ps}$, whereas the sodium charge distribution is less affected by the dissociation. A further interesting finding is the rather constant $\mathrm{NaCl}$ distance between 4.2 and 4.4 ps amounting to approximately $5 \AA$. Here, the system stabilizes itself in the form of a solvent separated ion pair.

The right side of Fig. 7 depicts the structure of the simulation at 4.3 ps. At that moment the system passes the intermediate, solvent separated ion pair phase (4.2-4.4 ps). It can be observed that one water molecule coordinates to both ions, thus being part of the first hydration shells of $\mathrm{Na}^{+}$as well as $\mathrm{Cl}^{-}$. It is oriented with one $\mathrm{H}$-atom pointing at $\mathrm{Cl}^{-}$and its O-atom towards $\mathrm{Na}^{+}$. A similar behaviour was also observed in the chloride-centered simulation. Unfortunately, this solvent separated intermediate was not sufficiently enduring and hence it was not possible to further investigate its structure and dynamical behavior in this simulation. At $4.5 \mathrm{ps}$ chloride entered the MM-region (see $\mathrm{Cl}^{-}$ charge of -1 a.u. in Fig. 7) and the simulation had to be considered complete. However, the observation of this solvent separated intermediate motivated the execution of another sodium-centered simulation employing different initial

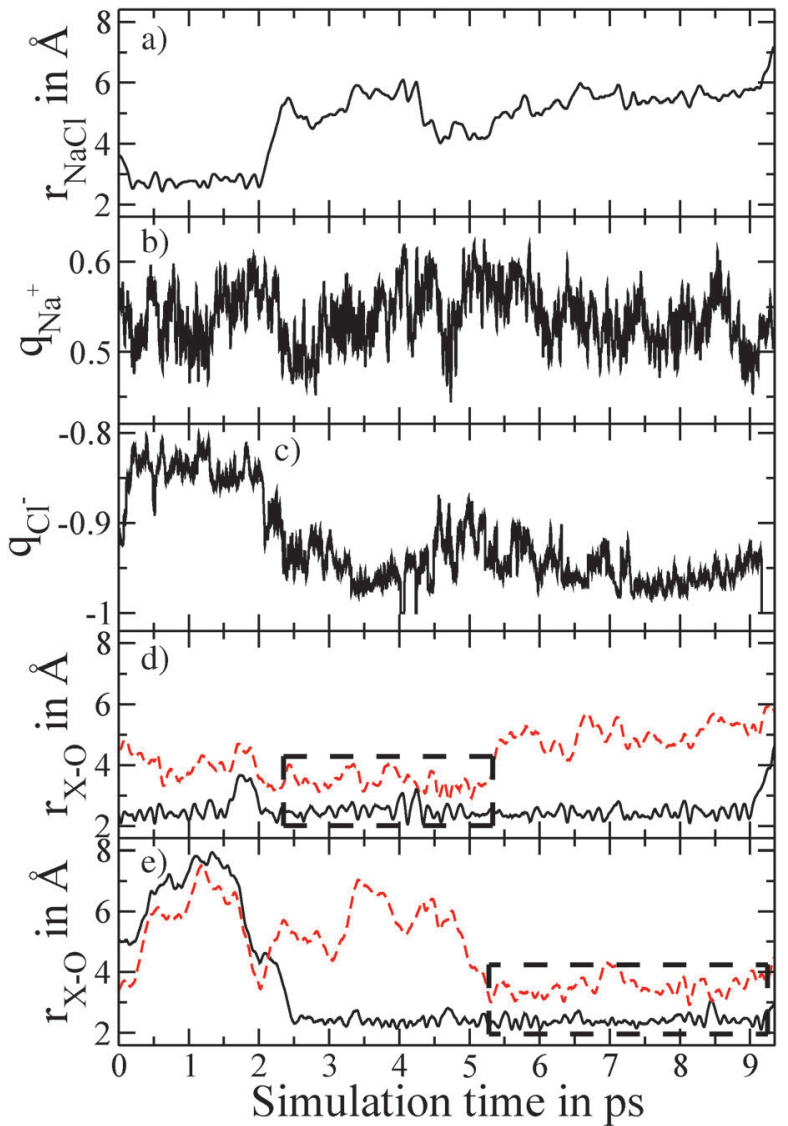

Fig. 8 (a) $\mathrm{NaCl}$ bond distance: (b) and (c) $\mathrm{Na}^{+}$and $\mathrm{Cl}^{-}$charge, respectively; (d) and (e) ion-oxygen distance plots of two different water molecules; the respective $\mathrm{Na}^{+}$-water distances are depicted as solid black lines, $\mathrm{Cl}^{-}$-water distances are depicted as dashed red lines. The boxes indicate time periods during which the respective water molecules were bound to both the ions.

conditions, to investigate whether the solvent separated ion pair can be observed for a longer period.

3.3.2 Simulation 2. The second simulation utilized a different starting geometry (see Section 3.1). In this run the intermediate state of a solvent separated ion pair was observed for a period of almost 7 ps (Fig. 8).

Analyzing the change in the $\mathrm{NaCl}$ bond distance and the charges on both ions over the course of the simulation, a number of coincidental events can be observed, like the pronounced drop in charge as soon as the system enters the solvent separated state. Similar to the first sodium-centered simulation, the chloride ion attempted twice to leave the QM zone ( $4.0 \mathrm{ps}$ and $4.3 \mathrm{ps}$ ), but returned within less than $0.1 \mathrm{ps}$. A third attempt occurring at about 9.2 ps was finally successful, thereby ending this simulation. The intermediate as well as the final transitions between the QM and MM zone happened accompanied by less pronounced effects on the charge of the sodium ion, confirming thereby that the cation's charge is mainly stabilized by hydration.

For a full description of all dynamical processes happening in the system and in order to compare them to the contact ion pair (chloride-centered simulation), the Mean Residence Times (MRTs) were calculated. For the results listed in Table 2 
Table 2 Results of the dynamical analysis of the exchange of water ligands in sodium's first and second and chloride's first hydration shell. Values for the contact ion pair are given in Table 1

\begin{tabular}{llll}
\hline & $\mathrm{Na}-\mathrm{O}$ (first shell) & $\mathrm{Na}-\mathrm{O}$ (second shell) & $\mathrm{Cl}-\mathrm{O}$ (first shell) \\
\hline$R_{\min } / \AA$ & 2.0 & 3.3 & 2.8 \\
$R_{\max } / \AA$ & 3.3 & 6.0 & 3.9 \\
$\mathrm{CN}_{\mathrm{av}}$ & 5.4 & 20.0 & 6.8 \\
$N_{\mathrm{ex}}$ & 11 & 80 & 110 \\
$\tau_{0.5} / \mathrm{ps}$ & 3.5 & 1.8 & 2.0 \\
$R_{\mathrm{ex}}$ & 3.2 & 4.7 & 4.6 \\
\hline
\end{tabular}
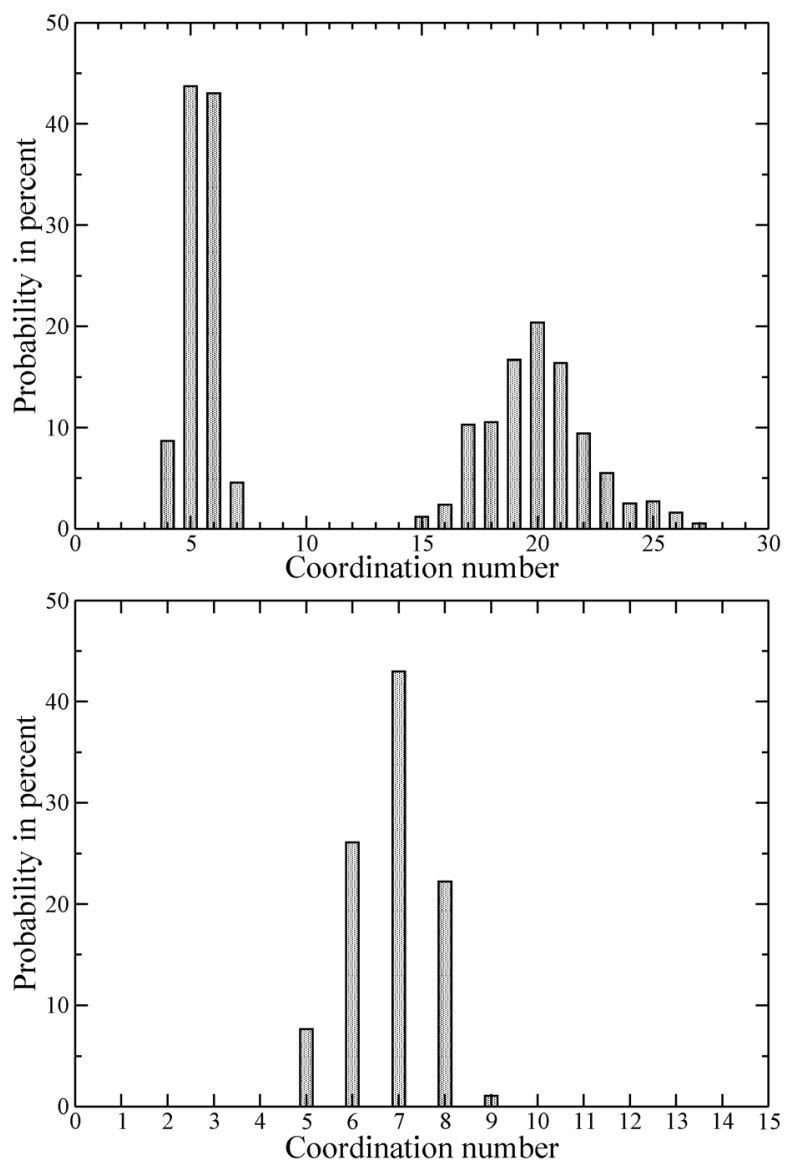

Fig. 9 Coordination Number Distributions (CNDs) of first shell oxygen around the $\mathrm{Na}^{+}$-ion (top) and the $\mathrm{Cl}^{-}$ion (bottom), respectively.

only those parts of the simulation were taken into account, in which the solvent separated ion pair is present (2 ps-9 ps). As before, $R_{\mathrm{ex}}$ is the reciprocal sustainability coefficient and defines how many attempts are needed on average to exchange one water molecule in the given hydration sphere.

The Coordination Number Distributions (CNDs) are depicted in Fig. 9.

Comparing these results with the ones obtained for the contact ion pair (Table 1 and Fig. 2), some distinct differences can be found. First, the average coordination number rises, because more space is available to accommodate water molecules. Another aspect that can be observed especially when looking at the sodium ion is the drop in successful exchanges associated with an increase in the MRT values. The vivid exchanges happening around the contact ion pair slow down once a solvent separated state is reached.

After analyzing the properties of the ions, the focus was shifted to the water molecule located in between them. Upon close examination of the observed data, it was found that an exchange of the ion-separating water molecule took place during the course of the simulation. A search algorithm enabled the identification of the associated water molecules. Over the whole course of the simulation, several water molecules entered and left the first hydration shell of either of the two ions, but only two solvent particles were part of the first hydration spheres of both ions at the same time. Fig. 10 depicts the $\mathrm{Na}^{+}$-water distances (solid lines) and the $\mathrm{Cl}^{-}$-water distances (dashed lines) of these two water molecules.

Fig. 8 shows the exchange of the intermediate water molecule during the dissociation of $\mathrm{NaCl}$. As depicted in Fig. 8d a water molecule positions itself between the two ions (framed area). At about 5.3 ps this water molecule leaves the hydration sphere of the chloride ion and another solvent molecule takes its place (framed area in Fig. 8e). The solvent separated species preserved for almost $7 \mathrm{ps}$, long enough to investigate the structural properties of this species. The obtained data depict an enlargement of the $\mathrm{NaCl}$ bond length from the average ion pair distance of $2.8 \AA$ - which is in good agreement with Fig. $3 \mathrm{~b}$ $(2.82 \AA)$ - to an extent of 5 to $6 \AA$. The plots depicted in Fig. 11 do not vary much from their contact ion pair counterparts (Fig. 4).

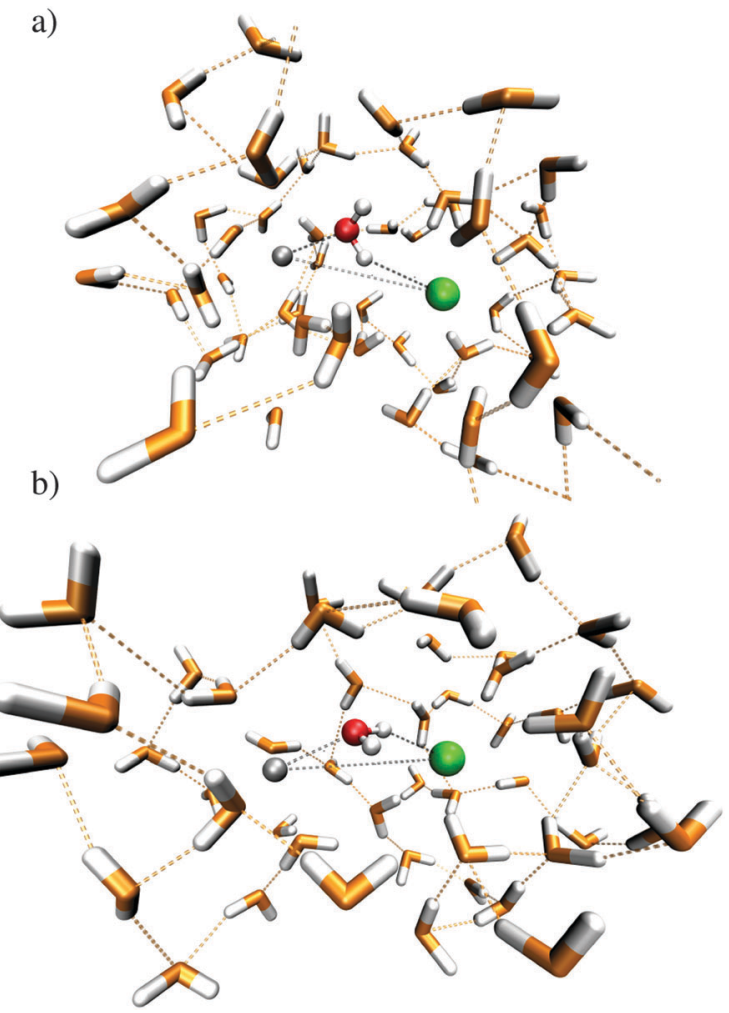

Fig. 10 (a) and (b) depict snapshots from the simulation at 2.4 and 5.8 ps, respectively, highlighting the two separating water molecules. 

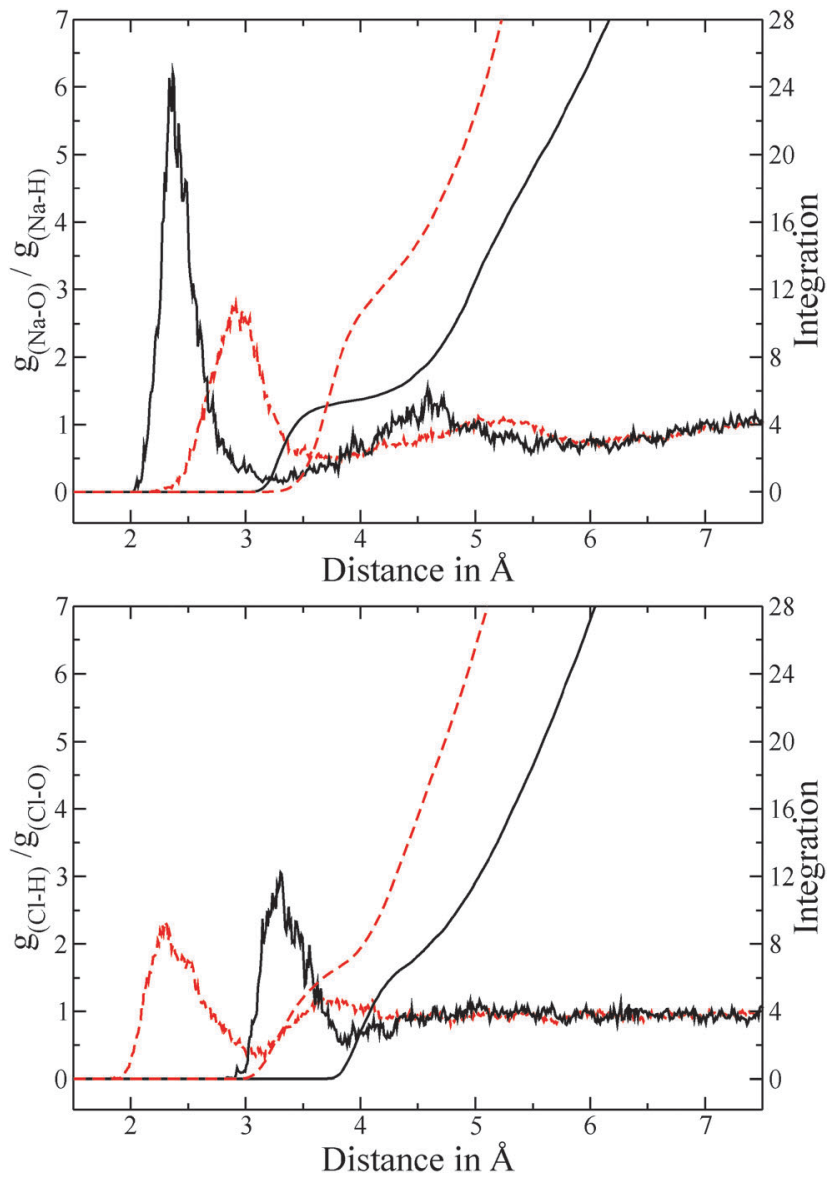

Fig. 11 Radial Distribution Functions (RDFs) during the solvent separated state; $\mathrm{Na}^{+}-\mathrm{H} / \mathrm{O}$ (top) and $\mathrm{Cl}^{-}-\mathrm{H} / \mathrm{O}$ (bottom); ion-O distances are depicted as solid black, ion-H distances as dashed red lines.

They basically show the same minima and maxima. The only major difference is the height of the peaks and the value of the integrals both are much higher for the solvent separated ion pair, as there is more space available to accommodate water molecules.

Another interesting aspect revealed by visualizing the trajectories is the orientation of the separating water molecule. For a major part of this simulation it was oriented with only one hydrogen atom binding to chloride, as illustrated in Figs. 10a and b.

\section{Conclusion and outlook}

This study of a dilute aqueous $\mathrm{NaCl}$ solution, based on results provided by QMCF-MD simulations, has obtained conclusive structural and dynamical information about the system, based on $a b$ initio QM treatment, and thus not subject to possible errors resulting from parametrisation, as occurring in classical MM simulations. The three different species postulated for such electrolyte solutions were observed. The interpretation of the dynamical data provided not only useful information about the $\mathrm{NaCl}$ bond strength in aqueous solutions, but it furthermore yielded valuable information about $\mathrm{NaCl}$ dissociation via a solvent separated intermediate, whose formation and structure we were able to elucidate on the basis of the obtained simulation results. In addition the evaluation of effective vibrational frequencies and the associated force constants provides important information about the strength and stability of various bonds, which are the basis for any equillibria in the course of the dissociation reaction. This information can further be utilized for the validation and further methodical development of empirical potentials.

\section{Acknowledgements}

The authors thank Thomas S. Hofer for his ideas and continuous support throughout this project.

\section{References}

1 S. Bouazizi, S. Nasr, N. Jaidane and M.-C. Bellissent-Funel, J. Phys. Chem. B, 2006, 110, 23515-23523.

2 R. Mancinelli, A. Botti, F. Bruni, M. A. Ricci and K. A. Soper, J. Phys. Chem. B, 2007, 111, 13570-13577.

3 M. Patra and M. Karttunen, J. Comput. Chem., 2004, 25, 678-689.

4 P. L. Geissler, C. Dellago and D. Chandler, J. Phys. Chem. B, 1999, 103, 3706-3710.

5 J. Timko, D. Bucher and S. Kuyucak, J. Chem. Phys., 2010, 132, 114510.

6 C. P. Petersen and M. S. Gordon, J. Phys. Chem. A, 1999, 103, 4162-4166.

7 E. Jardon-Valadez and M. E. Costas, J. Mol. Struct.: THEOCHEM, 2004, 677, 227-236.

8 S. Koneshan and J. C. Rasaiah, J. Chem. Phys., 2000, 113, 8125-8137.

9 C. J. Fennell, A. Bizjak, V. Vlachy and K. A. Dill, J. Phys. Chem. B, 2009, 113, 6782-6791.

10 Y. Yang, S. Meng and E. G. Wang, J. Phys.: Condens. Matter, 2006, 18, 10165-10177.

11 D. E. Smith and L. X. Dang, J. Chem. Phys., 1994, 100, 3757-3766.

12 S. Winstein, E. Clippinger, A. H. Fainberg and G. C. Robinson, J. Am. Chem. Soc., 1954, 76, 2597-2598.

13 M. K. Ghosh, S. Re, M. Feig, Y. Sugita and C. H. Choi, J. Phys. Chem. B, 2013, 117, 289-295.

14 B. M. Rode, T. S. Hofer, B. R. Randolf, C. F. Schwenk, D. Xenides and V. Vchirawongkwin, Theor. Chem. Acc., 2006, 115, 77-85.

15 T. S. Hofer, A. B. Pribil, B. R. Randolf and B. M. Rode, Adv. Quantum Chem., 2010, 59, 213-246.

16 T. S. Hofer, B. R. Randolf and B. M. Rode, in Solvation Effects on Molecules and Biomolecules, ed. S. Canuto, Springer, Heidelberg, 2008, vol. 6.

17 M. J. Field, P. A. Bash and M. Karplus, J. Comput. Chem., 1990, 11, 700-733.

18 D. Bakowies and W. Thiel, J. Phys. Chem., 1996, 100, 10580-10594. 
19 H. Lin and D. G. Truhlar, Theor. Chem. Acc., 2007, 117, 185-199.

20 A. K. Weiss and T. S. Hofer, RSC Adv., 2013, 3, 1606-1635.

21 M. Frisch, G. Trucks, H. Schlegel, G. Scuseria, M. Robb, J. Cheeseman, G. Scalmani, V. Barone, B. Mennucci, G. Petersson, H. Nakatsuji, M. Caricato, X. Li, H. Hratchian, A. Izmaylov, J. Bloino, G. Zheng, J. Sonnenberg, M. Hada, M. Ehara, K. Toyota, R. Fukudaand, J. Hasegawa, M. Ishida, T. Nakajima, Y. Honda, O. Kitao, H. Nakai, T. Vreven, J. A. Montgomery Jr., J. Peralta, J. J. Heyd, E. Brothers, K. Kudin, F. Ogliaro, M. Bearpark, V. Staroverov, R. Kobayashi, J. Normand, K. Raghavachari, A. Rendell, J. Burant, S. Iyengar, J. Tomasi, M. Cossi, N. Rega, J. Millamand, M. Klene, J. Knox, J. Cross, V. Bakken, C. Adamo, J. Jaramillo, R. Gomperts, R. Stratmann, O. Yazyev, A. Austin, R. Cammi, C. Pomelli, J. Ochterski, R. Martin, K. Morokuma, V. Zakrzewski, G. Voth, P. Salvador, J. Dannenberg, S. Dapprich, A. Daniels, O. Farkas, J. Foresman, J. Ortiz, J. Cioslowski and D. Fox, Gaussian, Inc., Wallingford CT, 2009.

22 C. C. Liew, H. Inomata and K. Arai, Fluid Phase Equilib., 1998, 144, 287-298.

23 K. Toukan and A. Rahman, Phys. Rev. B: Condens. Matter Mater. Phys., 1985, 31, 2643-2648.

24 H. Berendsen, J. Postma, W. van Gunsteren, A. DiNola and J. Haak, J. Chem. Phys., 1984, 81, 3684-3690.

25 J. Lennard-Jones, Proc. R. Soc. A, 1924, 106, 463-477.

26 W. L. Jorgensen and J. Tirado-Rives, J. Am. Chem. Soc., 1988, 110, 1657-1666.
27 P. Hariharan and J. Pople, Theor. Chem. Acc., 1973, 28, 213-222.

28 M. M. Francl, W. J. Petro, W. J. Hehre, J. S. Binkley, M. S. Gordon, D. J. DeFrees and J. A. Pople, J. Chem. Phys, 1982, 77, 3654-3665.

29 D. Xenides, B. R. Randolf and B. M. Rode, J. Chem. Phys., 2005, 122, 174506-174515.

30 J. Barker and R. Watts, Mol. Phys., 1973, 26, 789-792.

31 R. Watts, Mol. Phys., 1974, 28, 1069-1083.

32 T. S. Hofer, H. T. Tran, C. F. Schwenk and B. M. Rode, J. Comput. Chem., 2004, 25, 211-217.

33 T. Hogen-Esch and J. Smid, J. Am. Chem. Soc., 1966, 88, 307-318.

34 T. Hogen-Esch and J. Smid, J. Am. Chem. Soc., 1966, 88, 318-324.

35 S. Winstein, E. Clippinger, A. H. Fainberg, R. Heck and G. C. Robinson, J. Am. Chem. Soc., 1956, 78, 328-335.

36 E. Grunewald, Anal. Chem., 1954, 26, 1696-1701.

37 R. Mulliken, J. Chem. Phys., 1955, 23, 1833-1841.

38 R. Mulliken, J. Chem. Phys., 1962, 36, 3428-3440.

39 S. S. Azam, T. S. Hofer, B. R. Randolf and B. M. Rode, J. Phys. Chem. A, 2009, 113, 1827-1834.

40 A. Tongraar and B. Rode, Phys. Chem. Chem. Phys., 2003, 5, 357-362.

41 W. Barrett and W. Wallace, J. Am. Chem. Soc., 1954, 76, 366-369.

42 M. Straumanis and A. Ievins, Z. Phys., 1936, 102, 353-359.

43 A. K. Weiss, T. S. Hofer, B. R. Randolf, A. Bhattacharjee and B. M. Rode, Phys. Chem. Chem. Phys., 2011, 13, 12173-12185. 